\title{
Actualidades sobre el manejo endoscópico en complicaciones derivadas de la cirugía bariátrica
}

\author{
Martín Edgardo Rojano-Rodríguez*, Aida Monserrat Reséndiz-Barragán, Guadalupe Salinas-Cornejo y \\ Enrique Rentería-Palomo \\ Departamento de Cirugía Bariátrica, Hospital General Dr. Manuel Gea González, Ciudad de México, México
}

\begin{abstract}
Resumen
La obesidad es un problema de salud pública a nivel mundial, México es el segundo lugar a nivel mundial con obesidad en edad adulta y el primero en población pediátrica. Actualmente el procedimiento bariátrico más realizado es la manga gástrica laparoscópica y en segundo lugar la derivación gastroyeyunal. En nuestro país se reportaron, a la Federación Internacional de la Cirugía para la Obesidad y enfermedades metablicas(IFSO), 1881 durante el año 2018. A pesar de que la cirugía bariátrica ha demostrado ser un procedimiento con una amplia seguridad perioperatoria, no se encuentra exenta de complicaciones, un ejemplo es la fuga de manga gástrica, en la que el tratamiento endoscópico ya sea por medio de prótesis metálicas auto expandibles, drenaje interno con catéter doble cola de cochino, septotomía endoscópica, dilatación endoscópica así como la terapia de succión negativa endoscópica, deben ser una opción inicial por su baja morbilidad y su alta tasa de éxito. Para la coledocolitiasis en pacientes con derivación gastroyeyunal en Y de Roux, el tratamiento endoscópico en cualquiera de sus tres modalidades es el estándar de oro para el tratamiento de esta. En pacientes que presentan reganancia de peso la terapia de coagulación con argón plasma para reducir el diámetro de la gastroyeyuno anastomosis ha mostrado un buen perfil de éxito además de ser un procedimiento de mínima invasión. Así el manejo endoscópico intraluminal o de tercer espacio ha ganado una gran aceptación al demostrar un alto grado de éxito para resolver complicaciones con una menor tasa de efectos adversos.
\end{abstract}

Palabras clave: Obesidad. Procedimiento bariátrico. Tratamiento endoscópico. Manejo endoscópico intraluminal

En México, actualmente el segundo país más prevalente en sobrepeso y obesidad para población adulta siguiendo a Estados Unidos y el primer lugar con obesidad infantil a nivel mundial, tiene registrado según datos de la IFSO (International Federation for the Surgery of Obesity and Metabolic Disorders) que en el 2018 se realizaron cerca de 2000 procedimientos bariátricos en diversos centros quirúrugicos ${ }^{1}$.

En cuanto a la evaluación preoperatoria existen distintos puntos de vista sobre la realización de endoscopía preoperatoria entre las guías europeas, que la indican de manera rutinaria ${ }^{2}$, y las guías americanas que no la indican de manera estricta ${ }^{3}$. Una revisión sistemática realizada por Evans, et al. en la universidad de Washington demostró que en el $7.3 \%$ de los pacientes en quienes se realizó la endoscopía preoperatoria hubo cambio en el plan quirúrgico secundario a alguna alteración y que el costo por endoscopía diagnóstica fue de 22,142 dólares ${ }^{4}$; en contraste con nuestro medio el realizar estudios endoscópicos en los cuales se puedan diagnosticar patologías desde esofagitis hasta tumores, a un costo que no se equipara al costo de

\section{Correspondencia:}


estudios en Estados Unidos, nos debe llevar a considerar que el realizar este estudio muy útil a bajo costo debe de ser de rutina en todos nuestros pacientes.

Actualmente el procedimiento bariátrico más realizado a nivel mundial es la manga gástrica laparoscópica seguido del bypass gástrico laparoscópico. Las fugas luego de la gastrectomía vertical en manga es una de las complicaciones postoperatorias más temidas, debido a que usualmente el tratamiento es complejo y prolongado ${ }^{5}$. El sitio principal de fuga luego de una manga gástrica es en la región superior, justo debajo de la unión gastroesofágica. Una de las más nuevas maneras de tratamiento es la colocación de prótesis metálicos auto-expandibles, con una gran tasa de éxito ${ }^{6}$. Una de las principales complicaciones es la migración del mismo. Diogo, et al. presentaron un estudio multicéntrico en el que se observa un éxito de colocación del $100 \%$, y un éxito de cierre del $70 \%$, dentro de este estudio se compara un nuevo dispositivo completamente cubierto con un flap proximal largo, sin embargo no demostró tener una menor incidencia de migración y se asoció a un mayor número de complicaciones $^{6}$. El tratamiento de las fugas tardías y crónicas se basa en la dilatación endoscópica y de septotomías endoscópica, la cual fue introducida en 2009 , utilizando la igualdad de presión que se forma entre la cavidad del absceso y el estómago para permitir su drenaje en caso de fugas crónicas. Vargas, et al. de la clínica Mayo, mostraron el reporte de la experiencia que se ha tenido en su centro de referencia en casos complejos de fugas y fístulas refractarios a tratamientos habituales (drenaje percutáneo, prótesis). Incluyeron 28 pacientes, de los cuales en 18 se había realizado gastrectomía vertical, 9 se habían realizado bypass gástrico y 1 gastroplastia vertical en banda. $71 \%$ de fístulas y el $100 \%$ de las fugas se trataron exitosamente con técnicas endoscópicas combinadas con un promedio de tres procedimientos con una duración de 6.6 meses de tratamiento ${ }^{7}$. Debido a la reciente evidencia cada vez es más frecuente que el tratamiento de complicaciones en pacientes operados de cirugía bariátrica incluya terapias endoscópicas mínimamente invasivas y con excelentes resultados. En nuestro país Rojano, et al. presentó su experiencia en el tratamiento de 10 casos de colocación de prótesis metálicas auto-expandibles luego de fugas de manga gástrica, con un éxito de resolución del $100 \%$, dos pacientes presentaron úlcera por la prótesis y un paciente presentó estenosis, no se reportaron migraciones ${ }^{8}$.

Otra complicación, aunque poco frecuente luego del bypass gástrico, es la coledocolitiasis, la cual debido a la anatomía modificada, presenta un reto terapéutico para endoscopistas entrenados. De las modalidades terapéuticas que existen se encuentran la entero-CPRE (EA-CPRE), la cual se realiza con un video-enteroscopio, la segunda modalidad es realizar una fístula gastro-gástrica guiada por ultrasonido endoscópico y colocar una prótesis metálica entre ambas cavidades, realizando la CPRE de manera convencional, de la misma manera se puede realizar CPRE transgástrica (CPRE-LA) en sala de quirófano al realizar una gastrotomía laparoscópica e ingresar el duodenoscopio al estómago excluido para realizar el procedimiento. En un meta-análisis realizado por Ayoub comparando los resultados entre realizar CPRE-LA y EA-CPRE. Se incluyeron 26 estudios (14 CPRE-LA y 12 EA-CPRE) la tasa de éxito fue superior para la CPRE-LA 97.8\% contra $73.2 \%$ para el grupo de EA-CPRE aunque con mayor tiempo de procedimiento y de efectos adversos perioperatorios ${ }^{9}$. La realización de la CPRE transgástrica dirigida por ultrasonido (EDGE) ha ganado popularidad como una alternativa a las dos opciones previamente mencionadas. Usualmente se realiza con prótesis metálicas con lumen (LAMS), de $15 \mathrm{~mm}$. Un estudio realizado en la universidad de Virginia que incluyó 9 pacientes postoperados de bypass, con patología biliar obstructiva en los que se realizó EDGE con LAMS de $20 \mathrm{~mm}$ demostró que se tiene un tiempo de procedimiento menor así como menores efectos adversos que los reportados previamente en la literatura ${ }^{10}$. En la experiencia mexicana en el manejo endoscópico de complicaciones bariátricas presentado en la DDW, un grupo de 6 pacientes se les realizó CPRE transgástrica de manera laparoscópica, con un éxito de canulación del $100 \%$ y de resolución de la obstrucción de la vía biliar, solamente presentando efectos adversos menores en dos pacientes ${ }^{8}$.

En pacientes con reganancia de peso luego de bypass gástrico, el manejo endoscópico con reducción de tamaño del reservorio gástrico y de la gastroyeyuno anastomosis aparecen como una opción terapéutica para este problema. Fittipaldi comparó la coagulación con argón plasma contra un grupo placebo en el que solo se realizó endoscopía superior con sedación, los pacientes en que se realizó la reducción de la GYA presentaron una pérdida de peso de $15.02 \mathrm{Kg}+-9.3$ y en el grupo control hubo un aumento de peso de $0.5 \mathrm{Kg}$ +- $2.23^{11}$. Otro estudio realizado en el hospital Johns Hopkins en el que se utilizó ablación con crio-balón para la reducción tanto de la gastroyeyuno anastomosis como del tamaño del reservorio, se incluyeron 21 pacientes en los que se realizó el procedimiento y se 
realizó revisión endoscópica a las 8 y 16 semanas, en la primera revisión 11 pacientes requirieron nuevamente de la terapia y en la segunda solamente 4 , como resultado se observó que los pacientes presentaron una reducción de peso que solo correlacionó con la disminución del diámetro de la anastomosis y no para el tamaño del reservorio gástrico ${ }^{12}$. En cuanto a la reganancia de peso luego de manga gástrica se presentó un estudio multicéntrico entre la clínica Mayo y el hospital Johns Hopkins, utilizando sutura endoscópica de espesor total para crear nuevamente una manga más restrictiva, se incluyeron solo 9 pacientes con seguimiento a 9 meses con una pérdida del porcentaje del exceso de peso del 18.5\%+-10.3, reportando solo un paciente con estenosis que requirió dilatación endoscópica, aunque el tiempo de seguimiento fue muy corto, y se necesitan estudios con mayor número de pacientes y más duración, la sutura endoscópica es un tratamiento endoscópico seguro y efectivo acompañado de cambios en el estilo de vida y de terapia farmacológica $^{13}$. Un estudio realizado en nuestro país en el que se estudió la pérdida de peso comparando la reducción del diámetro de la GYA de manera endoscópica entre coagulación con argón plasma vs sobreclip OVESCO ${ }^{\circledR}$ demostró una pérdida mayor de peso con la terapia térmica $(17.6 \mathrm{Kg} \text { vs } 4.24 \mathrm{Kg})^{8}$.

\section{Conclusiones}

El manejo tradicional, abierto o laparoscópico, de las complicaciones que se presentan debido a la cirugía bariátrica suele acompañarse de morbilidad y mortalidad; el manejo endoscópico intraluminal o de tercer espacio ha ganado una gran aceptación al demostrar un alto grado de éxito para resolver estas complicaciones con una menor cantidad de efectos adversos. Todo cirujano bariatra debe conocer estas nuevas terapias endoscópicas, y así, ser el parte fundamental para el tratamiento de complicaciones en pacientes bariátricos y de esta forma realizarlo por cirujanos endoscopistas experimentados.

\section{Conflicto de intereses}

No existen conflictos de interés. Sin patrocinio de la industria.

\section{Bibliografía}

1. Angrisani L, Santonicola A, lovino P, Scopinaro N. IFSO Worldwide Survey 2016: Primary, Endoluminal, and Revisional Procedures. Obesity Surgery. 2018;28(12):3783-3794.

2. Sauerland S, Angrisani L, Tacchino R, Weiner R, Neugebauer E. Obesity surgery: Evidence-based guidelines of the European Association for Endoscopic Surgery (EAES). Surgical Endoscopy. 2004;19(2):200-221.

3. Evans J, Muthusamy V, Acosta R, Cash B. The role of endoscopy in the bariatric surgery patient. Gastrointestinal Endoscopy. 2015;81(5):1063-1072.

4. Evans G, Barker A, Kushnir V. High Cost For Low Yield: A Sytematic Review To Assess The Cost-Efectiveness Of Rutine Pre-Operative Egd Before Bariatric Surgery. Lecture presented at; 2019; Digestive Disease Week 2019, San Diego Californa USA.

5. Diaz R, Davalos G, Guerron D. Endoscopic Septotomy For The Treatment of Leak After Sleeve Gastrectomy. Lecture presented at; 2019; Digestive Disease Week 2019, San Diego, California.

6. de Moura D, de Moura E, Galvao M. Outcomes Of A Novel Bariatric Stent In The Management Of Leaks Following Sleeve Gastrectomy: A Multicenter Analisis. Lecture presented at; 2019; Digestive Disease Week 2019, San Diego, California.

7. Vargas E, Matar R, Abu Dayyeh B. Endoscopic Management Of Refractory And Complicated Post-Bariatrics Leaks And Fistulas. Lecture presented at; 2019; Digestive Disease Week 2019, San Diego, California.

8. Rojano M. Ensdoscopic Management For Complications Post-Bariatric Surgery: Experience In Mexico. Speech presented at; 2019; Digestive Disease Week 2019, San Diego, California.

9. Ayoub F, Brar T, Banerjee D. Laparoscopy-Assisted Versus Enteroscopy-Assisted Endoscopic Retrograde Cholangiopancreatography In Patients With Roux-En-Y Gastric Bypass: A Meta-Analysis. Speech presented at; 2019; Digestive Disease Week 2019, San Diego, California.

10. Hsueh W, Richard M, Abdelqader A. Eus-Directed Trasngastric Ercp With 20mm Lumen-Apposing Metal Stents In Patients With Roux-En-Y Gastric Bypass, Are We Closer To Perfection?. Speech presented at; 2019; Digestive Disease Week 2019, San Diego, California.

11. Fittipaldi-Fernandez R, Hoff A, Barrichello S, Rodrigues Guedes M. Outlet Roux-Y-Gastric Bypass Pouch Reduction Using Argon Plasma Coagulation To Treat Weight Regain: A Prospective Study Including A Sham Group. Speech presented at; 2019; Digestive Disease Week 2019, San Diego, California.

12. Chang S, Popov V, Thompson C. Endoscopin Balloon Dilatation For Treatment Of Sleeve Gastrectomy Stenosis: A Meta-Analysis. Speech presented at; 2019; Digestive Disease Week 2019, San Diego, California.

13. Vargas E, Nieto J, Abu Dayyeh B. Endoscopic Suturing For Weight Regain After Sleeve Gastrectomy: Multicenter Series. Lecture presented at; 2019. 